\title{
Peptide-based GIP receptor inhibition exhibits modest metabolic changes in mice when administered either alone or combined with GLP-1 agonism
}

\author{
Jason A. West ${ }^{1}$, Soumitra S. Ghosh ${ }^{2}$, David G. Parkes ${ }^{3}$, Anastasia Tsakmaki ${ }^{4}$, Rikke V. Grønlund ${ }^{5}$, Philip \\ J. Pedersen ${ }^{5}$, David Maggs ${ }^{1,6}$, Harith Rajagopalan ${ }^{1}$, Gavin A. Bewick ${ }^{4 *}$ \\ ${ }^{1}$ Fractyl Laboratories Inc, Lexington, MA, 02421, USA \\ ${ }^{2}$ Doon Associates LLC, San Diego, CA, 92129, USA \\ ${ }^{3}$ DGP Scientific Inc, San Diego, CA, 92014, USA \\ ${ }^{4}$ Diabetes Research Group, School of Life Course Sciences, Faculty of Life Science and Medicine, \\ King's College London, London, WC2R 2LS, England, UK \\ ${ }^{5}$ Gubra ApS, Hørsholm Kongevej 11B, Hørsholm, DK-2970, Denmark \\ ${ }^{6}$ Present address: Becton Dickinson Technologies \& Innovation, Raleigh-Durham, NC, 27709, USA \\ *Correspondence: Gavin A. Bewick, PhD, Diabetes Research Group, Guys Campus King's College \\ London, London SE1 1UL, UK Phone: +44 (0)20 7836 6274, gavin.bewick@kcl.ac.uk
}

\begin{abstract}
Objective: Combinatorial gut hormone therapy is one of the more promising strategies for identifying improved treatments for metabolic disease. Many approaches combine the established benefits of glucagon-like peptide-1 (GLP-1) agonism with one or more additional molecules with the aim of improving metabolic outcomes. Recent attention has been drawn to the glucose-dependent insulinotropic polypeptide (GIP) system due to compelling pre-clinical evidence describing the metabolic benefits of antagonising the GIP receptor (GIPR). We rationalised that benefit might be accrued from combining GIPR antagonism with GLP-1 agonism. To this end we investigated the metabolic effects of co-administration of previously reported peptide-based GIPR antagonists with the GLP-1 agonist liraglutide.
\end{abstract}

Methods: Two GIPR peptide antagonists, GIPA-1 (mouse GIP(3-30) $\mathrm{NH}_{2}$ ) and GIPA-2 ( ${ }^{\alpha} \mathrm{Ac}-\mathrm{K} 10[\gamma \mathrm{E} \gamma \mathrm{E}-$ C16]-Arg18-hGIP(5-42)), were pharmacologically characterised in vitro in an assay measuring cAMP production in CHO-K1 cells overexpressing the mouse GIPR. These peptides were then characterised in vivo in lean mice for their effect on oral glucose tolerance, as well as their ability to antagonize exogenous GIP action. Finally, a mouse model of diet-induced obesity (DIO) was used to investigate the potential metabolic benefits of chronic dosing of peptide-based GIPR antagonists, alone or in combination with liraglutide.

Results: In vitro, both GIPR peptides exhibited potent antagonistic properties, with GIPA-2 being the more potent of the two. Acute in vivo administration of GIPA-1 during an oral glucose tolerance test (OGTT) had negligible effects on glucose tolerance and circulated insulin in lean mice. In contrast, GIPA-2 impaired glucose tolerance and attenuated circulating insulin levels, with offsetting effects on glycemia noted with co-administration with exogenous mouse GIP, suggesting true antagonism via GIPA-2 at the GIP receptor. Chronic administration studies in a DIO mouse model showed expected effects of GLP-1 agonism (via liraglutide), lowering food intake, body weight, fasting blood glucose and plasma insulin concentrations while improving glucose sensitivity, whereas delivery of either GIPR antagonist alone had negligible effects on these parameters. Interestingly, chronic dual therapy with the GIPR antagonists and GLP-1 showed separation from single intervention arms 
though augmented insulin sensitizing effects (modestly lowering insulin and HOMA-IR) and lowering plasmas triglycerides and free-fatty acids, with more notable effects observed with GIPA-1 compared to GIPA-2.

Conclusion: We conclude that, in contrast to the well-documented effects of GLP-1R agonism, systemic administration of peptide-based GIPR antagonists demonstrate minimal benefit on metabolic parameters in DIO mice, exhibiting no major effects on body weight, food intake and glycaemic parameters. However, the co-administration of both a GIPR antagonist together with a GLP1 agonist uncovers interesting synergistic and beneficial effects on measures of insulin sensitivity, circulating lipids and certain adipose stores that seem influenced by the degree or nature of GIP receptor antagonism.

KEY WORDS: glucose-dependent insulinotropic polypeptide (GIP), antagonism, diet-induced obese (DIO) mice, glucagon-like peptide-1 (GLP-1), obesity, diabetes

\section{INTRODUCTION}

The global obesity and diabetes crises have prompted a concerted effort to identify effective novel treatments. Gut hormones exhibit well-characterised physiological roles ranging from effects on pancreatic islet hormone secretion, glucose concentrations, lipid metabolism, energy storage, gut motility, appetite, and body weight. These properties have drawn attention to their potential to treat metabolic disease, and the glucagon-like peptide-1 (GLP-1) drug class is now a well-established treatment for Type 2 diabetes (T2D) and obesity. Current investigational therapeutic strategies centre on designing drugs based on gut hormones that provide synergistic or additive effects in combination (e.g. GLP-1, oxyntomodulin, glucose-dependent insulinotropic polypeptide (GIP), amylin, peptide tyrosine tyrosine (PYY)), but questions remain regarding which combination of hormones will produce the most effective, safe and tolerated treatment for obesity and diabetes.

Two key glucoregulatory gut hormones, GIP and GLP-1, are secreted respectively by $\mathrm{K}$ cells, located predominately in the proximal small intestine, and by $L$ cells, most densely located in the distal small intestine and colon [1, 2]. Both hormones are secreted following nutrient intake and augment insulin secretion. Together they produce the incretin effect, a 2-3 fold increase in insulin production in response to oral glucose compared with the equivalent glucose dose administered intravenously [3,4], suggesting a combination of these two hormones could be beneficial for the treatment of T2D.

Indeed, GIP is the major incretin hormone in healthy individuals, responsible for around $70 \%$ of the effect [5]. However, individuals with T2D exhibit an impaired GIP insulinotropic effect [6] and increased fasting plasma GIP concentrations [7]. In contrast, administration of exogenous GLP-1 results in normalization of fasting hyperglycaemia in T2D patients [8]. Moreover, GLP-1 is a potent inhibitor of appetite, food intake [9] and glucagon secretion [10]. As a result, GLP-1 monotherapy is a key therapeutic modality for T2D therapy. Six different GLP-1 receptor (GLP-1R) agonists in either short- or long-acting forms are licensed in Europe and the USA for the management of T2D [11, 12].

Despite the introduction of GLP-1 agonists, and more recently, Sodium-Glucose Cotransporter-2 (SGLT2) inhibitors, into the T2D treatment paradigm, well-controlled diabetes management remains elusive and many patients progress to insulin therapies. Weight-loss surgeries like Roux-en-Y gastric bypass (RYGB) and vertical sleeve gastrectomy are the only treatments associated with T2D 
remission [13-18]. Among the proposed mechanisms contributing to the metabolic benefits of RYGB are alterations in gut hormone secretion and sensitivity [19]. For example, post-prandial plasma GLP1 levels dramatically increase after RYGB surgery [20], as GLP-1 secretion is increased in the distal regions of the small intestine due to increased nutrient delivery [21]. However, contradictory reports regarding GIP secretion following RYGB exist; some report a reduction in GIP [22, 23], others no alteration [24] whilst others an increase in secretion [25].

Growing evidence suggests that GIP may act as an obesogenic factor under certain circumstances, further complicating rational design of combination therapies [26]. The GIP receptor (GIPR) is expressed in the pancreas but is also found in adipocytes [27] and brain [28], which are important regulators of body weight. GIP increases the activity of lipoprotein lipase [29], elevating triglyceride accumulation in fat tissue [30] and inhibiting lipolysis [31, 32]. Moreover, in healthy individuals, GIPR has been identified in multiple genome wide association studies (GWAS) as a contributing factor to obesity in European populations [33-37]. Obesogenic diets cause hyperplasia of $K$ cells and increase their density, driving elevated circulating GIP concentrations [38, 39]. Finally, GIP, K cell, and GIPR knockout mice are resistant to high-fat diet (HFD)-induced obesity and insulin resistance [40-42]. Together the evidence that GIP plays a pathophysiological role in the development of obesity and insulin resistance warrants further investigation and has led to discovery programs focused on blocking GIP activity [26, 31, 43]. Several different GIPR antagonists have been identified including vaccines against GIP [44, 45], GIP neutralising antibodies [46], antibodies against the GIPR $[47,48]$ and antagonist peptides [49-52] (for a detailed summary of these studies see [43]). However, pharmacological approaches have not yielded satisfactory data and have been controversial.

A monoclonal GIP neutralising antibody prevented weight gain independently of food intake in a mouse model of diet-induced obesity (DIO), reduced fasting insulin concentrations and improved intraperitoneal glucose tolerance [46]. Gipg013, another GIPR neutralizing antibody that exhibits equally high binding potencies against the mouse, rat, dog, and human GIPRs was developed by Ravn et al. [47]. Peripheral administration of gipg013 to obese mice prevented body weight gain [53], and when administrated centrally, reduced body weight and adiposity, likely through a leptin sensitizing mechanism [53]. In a second study, gipg103 reduced food intake and weight gain in highfat fed mice, and improved glucose tolerance [54]. However, weight loss was not enhanced following coadministration of gipg103 with a GLP-1 agonist. Surprisingly, the combined antagonism of GIPR and GLP-1R produced additional protection against DIO [54]. In one of the most promising studies, Killion et al. demonstrated that peripheral administration of murine anti-GIPR antibody prevented DIO in mice and was associated with reduced food intake, improved metabolism and decreased resting respiratory exchange ratio [48]. Excitingly, similar findings were observed in obese nonhuman primates (NHPs) utilizing an anti-human GIPR antibody [48]. Additionally, enhanced weight loss was observed in both mice and NHPs when anti-GIPR antibodies were co-administered with GLP-1R agonists [48]. However, GIPR antagonism did not result in improved glucose tolerance.

Further controversy is evident with peptide based GIPR antagonists. GIP(3-30) $\mathrm{NH}_{2}$, an Nterminally truncated form of GIP has been described as a potent peptide-based antagonist [50, 55, 56]. Sub-chronic dosing of rat GIP(3-30) $\mathrm{NH}_{2}$ increased body weight, fat mass, triglycerides, LPL, and leptin levels in rats fed normal chow [57]. A fatty-acylated, N-terminally truncated GIP analogue with high in vitro antagonism potency for mouse GIPR had no effect on body weight whether dosed alone or in combination with liraglutide in DIO mice [58]. In contrast, a palmitoyl variant of Pro ${ }^{3} \mathrm{GIP}(3-30)$ with a C-terminal extension enhanced insulin sensitivity and reduced body weight as well as circulating glucose and insulin in DIO mice [49]. 
Previous studies have demonstrated that GIPR blockade increases sensitivity to GLP-1R agonism at least at the level of pancreatic beta cells [59,60]. Killion et al. revealed chronic antagonism of GIPR enhanced GLP-1R agonist-driven weight loss [48], even though similar effects were not reproduced with gipg103 antibody [54]. In addition to these studies, there is an additional body of literature supporting GIPR agonism and not antagonism as a therapeutic strategy for T2D [26, 6163], and clinical testing is ongoing for a dual GIPR/GLP-1R agonist peptide [64, 65]. Given the conflicting results surrounding the co-targeting of GIP and GLP-1 signalling pathways, we investigated the metabolic benefits of co-administration of peptide-based GIPR antagonists with a GLP-1 agonist, liraglutide. We used two GIPR antagonists; mouse GIP(3-30)NH $\mathrm{NH}_{2}$ (GIPA-1) as well as $\mathrm{N}^{\alpha} \mathrm{Ac}-\mathrm{K} 10[\gamma \mathrm{F} \gamma \mathrm{E}-\mathrm{C} 16]-\mathrm{Arg} 18-\mathrm{hGIP}(5-42)$ (GIPA-2)[58]. In vitro functional activities of the peptides were confirmed before the metabolic consequences of acute and chronic dosing in mice with or without the GLP-1R agonist liraglutide were determined. While GLP-1 agonism via liraglutide demonstrated the expected pharmacodynamic actions with chronic treatment of DIO mice, including weight loss, reduced food intake and reduced fasting blood glucose as well as improved insulin sensitivity, the validated GIPR peptide antagonists either alone or in combination showed modest effects on insulin sensitivity and adiposity in this study, underscoring the complex and multifaceted control of glucose homeostasis and metabolism in vivo.

\section{MATERIALS AND METHODS}

\subsection{Peptides}

Two GIPR antagonist peptides were manufactured by CPC Scientific Inc. (Sunnyvale, CA, USA):

GIPA-1 (mouse GIP(3-30) $\mathrm{NH}_{2}$ ) was selected for investigation in mouse studies as it is species specific. It has the sequence: Glu-Gly-Thr-Phe-Ile-Ser-Asp-Tyr-Ser-Ile-Ala-Met-Asp-Lys-Ile-Arg-Gln-Gln-AspPhe-Val-Asn-Trp-Leu-Leu-Ala-Gln-Arg- $\mathrm{NH}_{2}$

GIPA-2 ( ${ }^{\alpha}$ Ac-K10[үEүE-C16]-Arg18-hGIP(5-42) ) has been reported as a potent antagonist for mouse GIPR, with no cross-reactivity at mouse-derived GLP-1 or glucagon receptors [58]. It has the sequence: Ac-Thr-Phe-Ile-Ser-Asp-Lys(isoGlu-isoGlu-palm)-Ser-Ile-Ala-Met-Asp-Lys-Ile-Arg-Gln-GlnAsp-Phe-Val-Asn-Trp-Leu-Leu-Ala-Gln-Lys-Gly-Lys-Lys-Asn-Asp-Trp-Lys-His-Asn-Ile-Thr-Gln

Liraglutide was used for GLP-1 receptor agonism (Novo Nordisk, Bagsværd, Denmark).

Mouse GIP was sourced from Phoenix Pharmaceuticals (Burlingame, CA USA).

\subsection{In vitro receptor activity/ cAMP measurements}

Each peptide was tested for its ability to activate or inhibit the mouse GIP receptor using the cAMP Hunter ${ }^{\mathrm{TM}}$ CHO-K1 GIPR Gs cell line, in combination with the Hit Hunter ${ }^{\circledast}$ CAMP XP+ assay for determining CAMP, according to the manufacturer's instructions (Eurofins DiscoverX Corporation). Briefly, cells were plated in a 384 -well plate and incubated overnight at $37^{\circ} \mathrm{C} / 5 \% \mathrm{CO} 2$ to allow the cells to attach and grow. The next day, for agonist determination, cells were stimulated with increasing doses of either mouse GIP, GIPA-1, or GIPA-2 to induce a response. For antagonist determination, cells were pre-incubated with GIPA-1 or GIPA-2, followed by mouse GIP at the $\mathrm{EC}_{80}$ concentration of $14 \mathrm{pM}$. Following stimulation, signal was detected using the HitHunter cAMP Assay Detection Kit (Eurofins DiscoverX Corporation).

\subsection{Animals}


All animal experiments were performed according to the bioethical guidelines of Gubra (Hørsholm, Denmark) and in compliance with internationally accepted laboratory animal care and use principles with monitoring under the personal license 2017-15-0201-01378, which was issued by the Danish Animal Experimentation Council. Male C57BI/6JRj mice between 5-7 weeks old were purchased from Janvier Labs (La Rochelle, France). All mice were acclimated for at least one week before the initiation of all studies. Mice were kept at $22 \pm 2{ }^{\circ} \mathrm{C}$ on a 12:12-h light-dark cycle and had access to food (Altromin \#1324 [11\% fat, 24\% protein, 65\% carbohydrate], Brogaarden, Denmark) and water ad libitum unless noted otherwise. At the beginning of each study, mice were single housed, randomized, and evenly distributed into test groups based on body weight.

\subsection{Acute in vivo studies}

The acute effects of GIPA-1 and GIPA-2 on glucose homeostasis and insulin secretion were studied in 10-week old lean male C57BL/6JRj mice. Animals ( $n=8$ for each group) fasted for 4 hours, were dosed via subcutaneous injection with 1,5 or $10 \mathrm{mg} / \mathrm{kg}$ of either GIPA-1 15 minutes before oral glucose administration $(1.5 \mathrm{~g} / \mathrm{kg})$, or GIPA-2 60 minutes before oral glucose administration $(1.5 \mathrm{~g} / \mathrm{kg})$. Blood glucose was measured just before the administration of GIPA-1 or GIPA-2 and at 0, 15, 30 60, 120, 180 and $240 \mathrm{~min}$. Plasma insulin was measured at $15 \mathrm{~min}$.

The effects of GIPA-2 and mouse GIP alone or in combination were studied in 10-week old lean male $\mathrm{C} 57 \mathrm{BL} / 6 \mathrm{JRj}$ mice. Animals were fasted 4 hours prior to an intraperitoneal glucose bolus $(1.5 \mathrm{~g} / \mathrm{kg})$. Sixty minutes prior to glucose load animals received subcutaneous injection of $5 \mathrm{mg} / \mathrm{kg}$ GIPA-2, while animals receiving mouse GIP $(0.1 \mathrm{mg} / \mathrm{kg})$ were dosed subcutaneously 15 minutes prior to glucose load. Blood glucose was measured just before the administration of GIPA-2 and at 0, 15, 3060,120 , 180 and $240 \mathrm{~min}$. Plasma insulin was measured at 0, 15 and $240 \mathrm{~min}$.

\subsection{Chronic in vivo studies}

GIPA-1 study:

23-week-old male C57BL/6JRj mice ( $n=40$ ), fed a HFD (SSNIFF ${ }^{\oplus}$ diet $\# 12492[60 \%$ fat, 20\% protein, $20 \%$ carbohydrate], Soest, Germany) for 14 weeks, were distributed into 4 test groups and received daily either vehicle, liraglutide $(0.2 \mathrm{mg} / \mathrm{kg} / \mathrm{day}$ (a dose reliably known to elicit metabolic effects in multiple Gubra studies), GIPA-1 ( $4.5 \mathrm{mg} / \mathrm{kg} / \mathrm{day}$ ) or liraglutide + GIPA-1 for 4 weeks while still maintained on HFD. GIPA-1 was delivered using minipumps in order to achieve sufficient GIPR antagonism based on the properties of this peptide (Fig.2A). Liraglutide was dissolved in phosphatebuffered saline (PBS) plus $0.1 \%$ bovine serum albumin (BSA) (vehicle1) and GIPA-1 in DMSO/propylene glycol (50/50 volume per volume) (vehicle 2). Alzet $100 \mu \mathrm{L}$ minipumps (Alzet, Cupertino, CA, USA) were filled with GIPA-1 $(30 \mathrm{mg} / \mathrm{mL})$ or vehicle 2 and kept in $0.9 \%$ saline at $37^{\circ} \mathrm{C}$ overnight. The minipump was placed subcutaneously through a 1-cm long incision in the neck with the animal under isoflurane anesthesia. The incision was closed using a 6-0 Ethilon (Ethilon, Somerville, New Jersey, USA) suture, as previously described [66]. From day 0 (first dose) through day 28, mice received one of the following four treatments per day: (1) vehicle 1 (subcutaneous injection (SC), once daily (QD)) and continuous infusion of vehicle 2 via osmotic minipump; (2) 0.2 $\mathrm{mg} / \mathrm{kg}$ liraglutide, SC, QD) and continuous infusion of vehicle 2 via osmotic minipump; (3) vehicle 1 (SC, QD) and continuous infusion of GIPA-1 $(\sim 4.5 \mathrm{mg} / \mathrm{kg} / \mathrm{day} ; 56.8 \mathrm{nmol} / \mathrm{kg} / \mathrm{h})$ via osmotic minipump; and (4) liraglutide $(0.2 \mathrm{mg} / \mathrm{kg}$ liraglutide, SC, QD) plus continuous infusion of GIPA-1 ( 4.5 $\mathrm{mg} / \mathrm{kg} /$ day) via osmotic minipump. Dose levels of GIPA-1 were based on targeting plasma exposure levels sufficient to antagonise endogenous GIP and selected based on in vitro antagonism data. Fasted (4 hrs) blood glucose and insulin levels were measured on study day $-3,14$ and 27 . An oral 
glucose tolerance test (OGTT) was performed on day 21 of the study. On the day of the OGTT the fasted animals received their daily doses of vehicles, liraglutide and GIPA-1 thirty minutes before receiving a glucose bolus $(2 \mathrm{~g} / \mathrm{kg})$. Blood glucose was measured before their daily dosing and at 0 , 15, 30 60, 120, $180 \mathrm{~min}$ after glucose administration. Plasma insulin was measured at 0 and $15 \mathrm{~min}$. Whole body weight and food intake were measured throughout the study period. On the termination day (day 28), liver, epididymal fat depot, mesenteric fat depot, retroperitoneal fat depot and inguinal fat depot were dissected and weighted. Blood collected from the tail vein was used for determination of free fatty acids (FFAs) plasma concentration and blood collected via cardiac puncture was used for determination of terminal plasma triglyceride (TG) and total cholesterol (TC).

GIPA-2 study:

For studying the chronic effects of GIPA-2, male C57BL/6JRj mice $(n=40)$ were fed a HFD (SSNIFF ${ }^{\circledR}$ diet $\# 12492$ [60\% fat, $20 \%$ protein, $20 \%$ carbohydrate], Soest, Germany) for 43 weeks. DIO mice were randomized ( $n=10$ per group) based on baseline blood glucose and body weight. Liraglutide was dissolved in PBS plus $0.1 \%$ BSA (Vehicle1) and GIPA-2 in PBS (vehicle 2). Mice received one of the following four treatments daily for 28 days while still maintained on HFD: (1) vehicle 1 (SC, QD) and vehicle 2 (SC, QD); (2) $0.2 \mathrm{mg} / \mathrm{kg}$ liraglutide (SC, QD) and vehicle 2 ; (3) vehicle 1 (SC, QD) and 10 $\mathrm{mg} / \mathrm{kg}$ GIPA-2 (SC,QD); and (4) $0.2 \mathrm{mg} / \mathrm{kg}$ liraglutide (SC, QD) plus $10 \mathrm{mg} / \mathrm{kg}$ GIPA-2 (SC,QD). GIPA-2 dose levels were selected based on doses shown to sufficiently antagonize insulinotropic actions of exogenous GIP in acute IPGTT studies (Fig. 1J) adjusted for single daily dosing over the 4-week treatment period. Fasted ( $4 \mathrm{hrs}$ ) blood glucose and insulin were measured on study day $-4,10$ and 26. An OGTT was performed on day 17. On the day of the OGTT the fasted animals (4 hrs) received their daily doses of vehicles, liraglutide and GIPA-2 one hour before receiving a glucose bolus (2 $\mathrm{g} / \mathrm{kg}$ ). Blood glucose was measured before their daily dosing and at 0, 15, $3060,120,180$ and 240 min after glucose administration. An insulin tolerance test (ITT) was performed on day 24 . Fasting (4 hrs) mice received their daily doses of vehicles, liraglutide and GIPA-2 one hour before receiving insulin $(0.5 \mathrm{U} / \mathrm{kg}$, intraperitoneal injection). Blood glucose was measured before their daily dosing and at $0,15,3060,120,180 \mathrm{~min}$ after glucose administration. Similar measurements were performed as in the GIPA-1 study (body weight and food intake throughout the study; and liver and fat pad weight on termination day).

\subsection{Blood pharmacology}

Cardiac blood was sampled using a needle and vacuette ( $1 \mathrm{~mL} 25 \mathrm{G}$ needle) directly from the left ventricle. Tail blood samples were collected in $10 \mu \mathrm{L}$ heparinized glass capillary tubes, and directly transferred into an Eppendorf tube with a buffer and shaken before blood glucose measurement. Fasting blood glucose was measured using a BIOSEN c-Line glucose meter (EKF-Diagnostics, Barleben, Germany), insulin was measured using the Meso Scale Diagnostics platform, TG and TC were measured using commercial kits (Roche Diagnostics, Mannheim, Germany) on the Cobas C-501 autoanalyzer (Roche, Switzerland), and FFA levels were measured using a Wako Chemicals kit (Richmond, VA) on the Cobas C-501 autoanalyzer, all according to the manufacturers' instructions. Homeostatic model assessment of insulin resistance (HOMA-IR) was calculated using the following equation: [fasting serum glucose $(\mathrm{mmol} / \mathrm{L}) \times$ fasting serum insulin $(\mathrm{pmol} / \mathrm{L}) / 22.5$ ] to assess insulin resistance [67].

\subsection{Statistical analysis}

All statistical analyses were performed using Graph Pad Prism Version 8.1.2 (GraphPad Software). Results are expressed as mean \pm standard error of the mean (SEM). Statistical significance was 
evaluated using one-way or two-way analysis of variance (ANOVA), as appropriate, followed by Tukey's or Dunnett's post hoc test. Relevant tests are described in figure legends. A cut off of $p<$ 0.05 was used as a threshold for statistical significance.

\section{RESULTS}

\subsection{In vitro receptor activity of GIPR peptide antagonists}

We began by characterising the receptor-interaction properties of GIPA-1 and GIPA-2. Their agonist and antagonist potencies were measured via a CAMP production assay in CHO-K1 cells overexpressing the mouse GIPR. Neither GIPA-1 or GIPA-2 exhibited agonist properties against the mouse GIPR (Suppl. Fig. 1A and B). In antagonist assays, GIPA-2 was more potent in comparison to GIPA-1 at blocking mouse GIPR activation by mouse GIP for CAMP production with $\mathrm{IC}_{50}$ values of 3 $\mathrm{nM}$ and $483 \mathrm{nM}$ respectively (Fig. $1 \mathrm{~A}$ and $\mathrm{B}$ ).

\subsection{Acute in vivo effects of GIPR peptide antagonists in lean mice}

Having established both GIPA-1 and GIPA-2 as potent mouse GIPR antagonists in vitro, we aimed to determine their acute actions on oral glucose tolerance in lean mice.

Acute treatment with 1,5 or $10 \mathrm{mg} / \mathrm{kg}$ of GIPA-1 had no effect on glucose tolerance (Fig. $1 \mathrm{C}$ and D) or plasma insulin concentrations 15 minutes post oral glucose bolus (Fig. 1G). In contrast, delivery of the more potent GIPA-2 produced a dose dependent increase in glucose area under the curve (AUC) (Fig. 1E and F). This was associated with an inhibition of glucose stimulated insulin secretion as measured by plasma insulin concentrations $15 \mathrm{~min}$ post glucose bolus (Fig. 1G).

To determine if GIPA-2's effects on glucose tolerance were solely driven by its ability to block mouse GIPR signalling, we co-administered GIPA-2 with mouse GIP during an intraperitoneal glucose tolerance test (IPGTT). As expected, acute treatment with mouse GIP significantly improved glucose tolerance, whilst GIPA-2 significantly decreased glucose tolerance (Fig. $1 \mathrm{H}$ and I). A robust increase in plasma insulin was observed 15 minutes after mouse GIP administration, just prior to glucose bolus (0 min timepoint). This effect was not observed at the 15 and $240 \mathrm{~min}$ timepoints (Fig. 1J). Importantly, combined treatment with GIPA-2 and mouse GIP resulted in attenuation of glucose tolerance (Fig. $1 \mathrm{H}$ and I) compared to mouse GIP treatment and an inhibition of mouse GIP stimulation of insulin secretion (Fig. 1J), demonstrating GIPA-2 to be an effective antagonist of exogenous GIP actions in vivo.

3.3 Chronic in vivo effects of GIPR peptide antagonists alone and in combination with GLP-1R agonist in DIO mice

We used a mouse model of DIO and insulin resistance to investigate the potential chronic metabolic benefits of GIPA-1 and GIPA-2 administration, alone or in combination with the GLP-1 receptor agonist liraglutide. DIO mice were treated for 28 days and food intake, body weight, and fasting glucose and insulin concentrations were measured at regular intervals (Fig. 2A). GIPA-1 and GIPA-2 studies were performed in chronological order but are presented side by side for comparison purposes.

Chronic administration of GIPA-1 or GIPA-2 alone had no effect on absolute body weight (Fig.2B and $C$ ) or cumulative food intake (Fig. 2D and E) compared with vehicle control. As expected, 
liraglutide reduced both body weight and food intake, but no additive effect was observed by combining liraglutide with either of the antagonists on these parameters.

Fasting blood glucose and plasma insulin concentrations were similar between groups at the beginning of the studies (Fig. 2F-I). In agreement with our acute dosing data, chronic dosing of GIPA1 alone did not alter fasting blood glucose at any time point (Fig. 2F), whilst liraglutide produced significant reductions for the duration of the study. In combination, liraglutide plus GIPA-1 also reduced fasting blood glucose but the combination was only significantly better at reducing blood glucose than liraglutide alone on day 14 . Associated plasma insulin was reduced in both the liraglutide and combination groups. This was significant for the combination, but not for liraglutide monotherapy (Fig. 2H).

GIPA-2 modestly reduced fasting glucose concentrations only being significant on day 10 (Fig. $2 \mathrm{G})$. In combination with liraglutide, GIPA-2 had no additive effect on the ability of liraglutide to lower fasting glucose concentration (Fig. 2G). Fasting insulin concentrations were not significantly different between groups (Fig. 2I).

Interestingly, the one area where the combinatorial effects of pharmacologic inhibition of GIPR and GLP-1R agonism showed some separation from the single intervention arm (GLP-1 alone) was with regard to plasma insulin levels and calculations of homeostatic model assessment of insulin resistance (HOMA-IR). On days 14 and 27 of the GIPA-1 study, GIPA-1 combined with liraglutide reduced HFD-induced hyperinsulinemia and insulin resistance (Fig. 2J). HOMA-IR was determined on day 10 and 26 of the GIPA-2 study (Fig. $2 \mathrm{~K}$ ). On day 10 , only the combination therapy showed a reduction in insulin resistance, whereas on day 26 both liraglutide monotherapy and combination therapy demonstrated a similar reduction in HOMA-IR compared to vehicle control.

The effect of GIPR antagonism on oral glucose tolerance was tested mid-study. Whereas GIPA-1 and GIPA-2 monotherapy resulted in elevated glucose excursions, their administration with liraglutide did not adversely impact the beneficial glucose lowering effects of liraglutide (Fig. 3A and B, E and F). In the GIPA-1 study, baseline blood glucose (-30 min) (Fig. 3A) was lower in the liraglutide group and in the combination group, in line with our previous results (Fig. 2F), but this effect was not evident for GIPA-2 (Fig. 3E).

Fasting insulin concentrations tracked the glucose trend for GIPA-1, with no effect compared to vehicle control when given alone, and no additional reduction of liraglutide-induced decrement in insulin at time 0 of the OGTT (Fig. 3C). A similar non-significant pattern of results was apparent at 15 minutes post glucose administration (Fig. 3D).

In the GIPA-2 study, we assessed insulin sensitivity using an ITT protocol on day 24. GIPA-2 monotherapy had no effect on insulin sensitivity and when delivered in combination with liraglutide appeared to negatively impact the improved insulin sensitivity produced by liraglutide (Fig. 3G and H).

Finally, we studied the chronic effects of GIPR antagonism on circulating lipids (total cholesterol (TC), triglycerides (TG) and free fatty acids (FFA)) and white adipose depots (including epididymal, mesenteric, inguinal, and retroperitoneal fat). We also accounted for fat deposition in key internal organs through measures of pancreas, kidney, duodenum, jejunum-ileum and liver weight. Notably, the combination of GIPR antagonism via GIPA-1 and GLP-1 agonism had effects to lower plasma TG (Fig 4A) plasma FFA (Fig 4C), and to cause a reduction in epididymal fat mass (Fig. 4G) GIPA-2 showed similar reduction in epididymal fata mass on combination with liraglutide (Fig. $4 \mathrm{H}$ ) when compared with single intervention arms. While this is consistent with reports of GIP's role in the 
regulation of adiposity, we did not observe similar and consistent effects of GIPR antagonism on circulating lipids and other fat depots. Monotherapy with either of the GIPR antagonists or liraglutide had no effect on either plasma TG or concentrations of circulating FFA (Fig. 4 A-D). Liraglutide alone significantly reduced circulating TC and liver TG concentrations but GIPR antagonism did not (Fig. 4E and F and Suppl. Fig. 2A and B). There was no impact on liver weight in any group (Suppl. Fig. $2 C$ and $D$ ). While the combination elicited a significant impact on TC concentrations (Fig. 4E and F) and liver TG (Suppl. Fig. 2A and2B), this effect was not superior to that of liraglutide monotherapy (Fig. 4E).. Mesenteric fat weight was reduced by combination therapy in the GIPA-1 study, but no additive effect was apparent (Fig. 4I). In the GIPA-2 study, mesenteric fat weight was unaffected by any treatment (Fig. 4J). Retroperitoneal fat weight was reduced in the GIPA-1 study in both the liraglutide and combination groups but was again unaffected in the GIPA-2 study (Suppl. Fig. 2E and F). There were no differences on inguinal fat weight between groups in either study (Suppl. Fig. 2G and H).

\section{DISCUSSION}

The identification of novel treatments to combat the burgeoning obesity and diabetes crisis is an urgent health priority. Considerable attention has focused on the physiological actions of gut hormones to control blood glucose, food intake and adiposity. The GLP-1 drug class has emerged as one of the most efficacious and widely-used treatment for diabetes in addition to demonstrated effects for weight loss $[68,69]$. However, T2D patients treated with GLP-1 mimetics still experience disease progression eventually requiring insulin therapy, and extensive efforts to improve the use of gut hormone-based therapy are focused on the rational design of combinations that are superior to monotherapy due to synergistic or additive effects on glucose control and weight loss. However, gut hormones as a therapeutic class often show undesirable side effects including nausea, vomiting, and diarrhoea, and for GLP-1 mimetics and investigational GLP-1 combination therapies, nausea can lead to discontinuation of therapy $[65,70]$.

The GLP-1-agonist-GIPR-antagonist combination therapy reported in the present study was born from evidence of compromised gut-hormone function in obesity and diabetes as well as their postulated role in driving the beneficial metabolic effects following bariatric surgery. Indeed, GLP-1 secretion is enhanced after bariatric surgery [20] which is thought to be a key component for the resolution of diabetes. In contrast, the controversial role of the GIP signalling pathway in dysmetabolic states has led to the pursuit of both agonist and antagonist approaches [26, 71]. There is a compelling body of evidence suggesting that antagonism of this system can produce beneficial effects on adiposity and insulin sensitivity $[46,48,49,53,54]$. The combination of the reported attributes afforded by GIPR antagonism as well as GLP-1's well-established metabolic benefits offered a rationale for investigating this dual therapy in the current study. To explore the potential of this combination, we selected two distinct and pharmacologically-characterised GIPR antagonists for evaluation, GIPA-1 (mouse GIP(3-30)NH ${ }_{2}$ ) and GIPA-2 ( ${ }^{\alpha} \mathrm{Ac}-\mathrm{K} 10[\gamma \mathrm{E} \gamma \mathrm{E}-\mathrm{C} 16]-\operatorname{Arg} 18-\mathrm{h} \mathrm{GIP}(5-42)$ ). Since the GIPR antagonist, (Pro3)-GIP, was originally characterized as an antagonist but was subsequently found to also possess partial agonist properties [72], we felt it prudent to characterise both the antagonist and agonist properties of these compounds. To our knowledge, no previous studies have determined the potency of mouse GIP(3-30) $\mathrm{NH}_{2}$ antagonism at the mouse GIPR. Sparre-Ulrich et al [55] have reported that in the presence of $10 \mathrm{pM}$ rat GIP(1-42), rat GIP(3-30) $\mathrm{NH}_{2}$ dose-dependently antagonized rat GIPR-stimulated cAMP accumulation with an estimated $\mathrm{EC}_{50}$ value 
of $118 \mathrm{nM}$. Further, rat GIP(3-30) $\mathrm{NH}_{2}$ exhibited a low-potency partial agonist profile at the rat GIPR $\left(E C_{50}>1 \mu \mathrm{M}\right)$. The human version of $\mathrm{GIP}(3-30) \mathrm{NH}_{2}$ has an antagonist $\mathrm{IC}_{50}$ of $11.8 \mathrm{nM}$ and and $\mathrm{K}_{\mathrm{i}}$ of $16.8 \mathrm{nM}$ respectively for human GIP-induced CAMP production in HEK293 cells and COS-7 cells transiently transfected with the human GIPR and does not exhibit agonist tendencies. In our hands mouse GIP(3-30) $\mathrm{NH}_{2}$ (GIPA-1) did not display agonist behaviour and inhibited mouse GIP-induced activation of mouse GIPR with an IC 50 of $483 \mathrm{nM}$, and therefore mouse GIP(3-30) $\mathrm{NH}_{2}$ may be a less potent antagonist compared to the rat and human sequences, although GIPR receptor differences could account for the observed effects. GIPA-2 was a significantly more potent antagonist at mouse GIPR with an IC 50 of $3 \mathrm{nM}$ and with no evidence of agonism, confirming the findings of Mroz et al. [58].

In agreement with our in vitro data, GIPA-2 acutely blocked the endogenous GIP incretin effect, impaired glucose tolerance, and inhibited the improved glucose tolerance elicited by exogenously administered native GIP. In contrast, GIPA-1 had no effect on glucose tolerance. We suspected the apparent lack of effect could be due to the short circulating half-life of GIPA-1; the human peptide sequence has a plasma elimination half-life of $7.7 \pm 1.4$ minutes in humans [56]. Given the strong in vitro pharmacological data and our interest in other metabolic parameters such as adiposity and insulin sensitivity we proceeded with GIPA-1 in our long term DIO studies but delivered the compound using an osmotic minipump to maintain sufficient plasma concentrations for durable GIPR antagonism given its in vitro activity. For GIPA-2 we used QD subcutaneous injection based on its extended plasma half-life and in vivo duration of action, as previously described [58].

This is the first description of chronic delivery of mouse GIP(3-30) $\mathrm{NH}_{2}$ (GIPA-1) to DIO mice, alone or in combination with liraglutide. GIPA-1 did not alter body weight or dietary consumption. However, its combination with liraglutide led to improvements in metabolic parameters including reductions in epididymal fat weight, plasma FFA, and HOMA-IR, as well as showing trends in reduced total TG and TC concentrations, when compared with liraglutide monotherapy. Of note, when administered alone, GIPA-1 negatively impacted glucose control during a glucose challenge but had no effect when administered together with liraglutide. Taken together, our data indicate that inhibition of GIPR activity with GIPA-1 in combination with GLP-1R agonism is associated with modest metabolic benefits including reduced insulin concentrations and improved insulin sensitivity without compromising glucose control.

Similarly to GIPA-1, GIPA-2 monotherapy had no effect on body weight or dietary consumption in agreement with previous reports, albeit delivered at a lower dose and shorter time period than in our study [58]. Co-administration of GIPA-2 with liraglutide significantly reduced epididymal fat weight and HOMA-IR and tended to reduce plasma FFA, when compared with liraglutide alone. Importantly, despite having a potentiating effect on glucose excursion during an OGTT when given alone, GIPA-2 did not inhibit liraglutide's beneficial glucose-lowering effect when administered in combination, mirroring our GIPA-1 data. The effect of treatments on different fat pad depots was generally mixed, and combination therapy provided no extra benefit over liraglutide apart from reducing epididymal fat pad weight.

Taken together our data suggest that combined GLP-1 agonism and peptide based GIP antagonism produces relatively modest metabolic benefits over GLP-1 given alone, highlighted by our results showing moderate improvements in fasting glucose, HOMA-IR and the weight of some fat depots due to combination therapy. However, these benefits are not robust with the antagonists and methodologies used in the current study. Further optimised peptide antagonists may produce stronger effects, more akin to those seen with GIPR antibody approaches. However, the question of 
how best to target the GIP system remains enigmatic. Why do both GIP agonist and antagonist approaches potentially enhance the metabolic improvements seen with GLP-1 mimetics? Early clinical results for a dual agonist peptide for both the GLP-1 and GIP receptors support a path forward for GIPR agonist approaches for treating T2D and/or obesity [26, 61-63]; however, preclinical data from multiple independent studies also validate a clinical strategy that employs a GIPR antagonist [46, 48, 49, 53, 54]. A recent review by Holst elegantly describes this conundrum and presented several possible scenarios focused around GIP receptor desensitisation, internalisation and post receptor signalling recruitment leading to GIP resistance [71]. Importantly, GIP antagonists appear able to restore the cell surface expression of GIPR [73] and therefore possess the potential to restore endogenous GIP sensitivity, a function lost in T2DM. This has direct implications for our studies. The available GIPR antagonists likely display varied potencies for GIPR, and responsiveness to endogenous GIP, and the GIPR antibody strategy is presumably the most potent. However, the challenge remains; how to engineer the balance between pharmacological GIPR antagonism and enhanced endogenous GIP sensitivity to thereby generate a metabolic benefit, and can this balance be more precisely controlled? These are questions which will require further investigation and may result in GIPR antagonists which are more suited for deploying in combination with GLP-1 agonists.

\section{Figure legends:}

Figure 1: Effects of acute GIPR antagonism on glucose homeostasis and insulin secretion. GIPA-1 and GIPA-2 are antagonists of mouse GIPR. Plots (A and B) shows the in hibition of mouse GIPR by GIPA-1 (A) and GIPA-2(B) in CHO-K1 cells. IC 50 values are indicated on the plots. (C-J) Acute effects of GIPA-1 and GIPA-2 on glucose homeostasis and insulin secretion in lean mice. Oral glucose tolerance tests (C and $E$ ), the corresponding areas under the curve (AUC) (D and F) and insulin responses (I) following subcutaneous injections of vehicle, 1,5 and $10 \mathrm{mg} / \mathrm{kg}$ GIPA-1 (C and D) or vehicle, 1,5 and $10 \mathrm{mg} / \mathrm{kg}$ GIPA-2 (E and F) before an oral glucose administration ( $1.5 \mathrm{~g} / \mathrm{kg})$. (G, $\mathrm{H}$ and J) Acute effect of GIPA-2 alone or in combination with mouse GIP on glucose homeostasis and insulin secretion in lean mice. Intraperitoneal glucose tolerance test cute IPGTT $(G)$, the corresponding $A U C(H)$ and insulin response (J) following subcutaneous injections of vehicle, $0.1 \mathrm{mg} / \mathrm{kg}$ mouse GIP, $5 \mathrm{mg} / \mathrm{kg}$ GIPA-2, and combination of both. Group sizes are $n=8$, and data are represented as mean \pm SEM. Statistical analysis was calculated using two-way ANOVA with Tukey's post-hoc test (C, E, G and J) and one-way ANOVA with Dunnett's post-hoc test (D, F, H, I). *: p< 0.05, **: $\mathrm{p}<0.01, * * *: p<0.001, * * *$ : $\mathrm{p}<0.0001$ compared to vehicle.

Figure 2: Effects of chronic GIPR antagonism on body weight, food intake, and fasting blood glucose and insulin. (A) GIPA-1 and GIPA-2 chronic studies schematic. (B-K) Effects of chronic administration of GIPA-1 and GIPA-2 on body weight (B and C), food intake (D and E), fasting plasma glucose ( $F$ and $G$ ), fasting plasma insulin ( $H$ and $I$ ) and HOMA-IR ( $\mathrm{J}$ and $K$ ) in DIO mice. Group sizes are $n=10$, and data are represented as mean \pm SEM. Statistical analysis was calculated using two-way ANOVA with Tukey's post-hoc test and one-way ANOVA for AUC with Dunnett's post-hoc test. ${ }^{*}: p<0.05,{ }^{* *}: p<$ $0.01, * * *: p<0.001, * * * *$ : $<0.0001$ compared to vehicle.

Figure 3: Effects of GIPA-1 on oral glucose tolerance and insulin responses in DIO mice. Oral glucose tolerance test (A), the corresponding glucose AUC (B) and insulin responses at $0 \mathrm{~min}(\mathrm{C})$ and $30 \mathrm{~min}$ (D) after the glucose bolus, following administration of vehicle, $0.2 \mathrm{mg} / \mathrm{kg}$ liraglutide, $\sim 4.5 \mathrm{mg} / \mathrm{kg}$ GIPA-1 and combination of both for 21 days in DIO mice.(E-H) Effects on oral glucose tolerance test ( $E$ and $F$ ) on day 17, and insulin tolerance test ( $G$ and $H$ ) on day study 24 , following chronic administration of vehicle, $0.2 \mathrm{mg} / \mathrm{kg}$ liraglutide, $10 \mathrm{mg} / \mathrm{kg}$ GIPA-2 and combination of both in DIO 
mice. Group sizes are $n=10$, and data are represented as mean \pm SEM. Statistical analysis was calculated using two-way ANOVA with Tukey's post-hoc test and one-way ANOVA with Dunnett's post-hoc test. ${ }^{*}: \mathrm{p}<0.05, * *: \mathrm{p}<0.01, * * *: \mathrm{p}<0.001, * * * *: \mathrm{p}<0.0001$ compared to vehicle.

Figure 4: The effect of GIPR antagonism alone or in combination with liraglutide on lipid homeostasis in DIO mice. Effects of GIPA-1 and GIPA-2 on plasma TG (A and B), FFAs (C and D), TC ( E and F), epididymal fat weight ( $G$ and $H$ ) and mesenteric fat weight ( $I$ and $J$ ) following administration of vehicle, liraglutide, GIPR antagonist, and liraglutide in combination with the GIPR antagonist for 28 days in DIO mice. Group sizes are $n=10$, and data are represented as mean \pm SEM. Statistical analysis was calculated using one-way ANOVA with Dunnett's post-hoc test. ${ }^{*}: p<0.05,{ }^{* *}: p<0.01,{ }^{* * *}: p<$ $0.001, * * * *: p<0.0001$ compared to vehicle.

Supplementary Figure 1: Representative concentration response curves for stimulation of cAMP accumulation by GIPA-1 (A) and GIPA-2 (B) in CHO-K1 cells expressing mouse GIPR, showing that neither is acting as partial agonist.

Supplementary Figure 2: The effect of GIPR antagonists alone or in combination with liraglutide on lipid homeostasis in DIO mice. Effects of GIPA-1 and GIPA-2 on liver TG (A and B), liver weight (C and $D)$, retroperitoneal fat weight ( $E$ and $F$ ) and inguinal fat weight ( $G$ and $H$ ) following administration of vehicle, liraglutide, GIPR antagonist, and liraglutide in combination with the GIPR antagonist for 28 days in DIO mice. Group sizes are $n=10$, and data are represented as mean \pm SEM. Statistical analysis was calculated using one-way ANOVA with Dunnett's post-hoc test. ${ }^{*}: p<0.05,{ }^{* *}: p<0.01,{ }^{* * *}$ : $p<$ $0.001, * * * *: p<0.0001$ compared to vehicle.

\section{ACKNOWLEDGMENTS}

This study was supported by Fractyl Laboratories Inc., Lexington, MA. The authors would like to thank Jason Huang at Fractyl for project management support, Jay Caplan and Emily Cozzi at Fractyl for critical review of this manuscript, and Gitte Hansen, Mette Østergaard, Bidda Rolin, and Cecilia Ratner at Gubra for supporting rodent studies.

\section{AUTHOR CONTRIBUTIONS}

Conceptualization and methodology: J.A.W., S.S.G., D.G.P., P.J.P., D.M., and H.R.; Investigation: J.A.W., S.S.G., D.G.P, P.J.P, R.V.G., D.M., H.R.; Formal analysis and writing, review, and editing: J.A.W., S.S.G., D.G.P., A.T., D.M., H.R, G.A.B. All authors reviewed and critiqued the manuscript throughout the editorial process, approved the final manuscript draft submitted for publication, and agreed to be accountable for all aspects of the work, ensuring the accuracy and integrity of the publication.

\section{DECLARATION OF INTERESTS}

J.A.W. and H.R. and are employees and shareholders of Fractyl Laboratories Inc. S.S.G. and D.G.P. have received honorariums for consultancy from Fractyl Laboratories Inc. A.T. has received funding/grant support from the Juvenile Diabetes Research Foundation (JDRF). P.J.P. and R.V.G. are employees of Gubra ApS. D.M. is an ex-employee of Fractyl Laboratories Inc., is a current shareholder, and has received honorarium for consultancy from Fractyl Laboratories Inc. G.A.B. has received funding/grant support from the European Foundation for the Study of Diabetes and JDRF and honorarium for consultancy from Fractyl Laboratories Inc. 


\section{REFERENCES:}

1. Reimann, F. and F.M. Gribble, Mechanisms underlying glucose-dependent insulinotropic polypeptide and glucagon-like peptide-1 secretion. J Diabetes Investig, 2016. 7 Suppl 1: p. 13-9.

2. Gehart, H., et al., Identification of Enteroendocrine Regulators by Real-Time Single-Cell Differentiation Mapping. Cell, 2019. 176(5): p. 1158-1173 e16.

3. Nauck, M.A. and J.J. Meier, Incretin hormones: Their role in health and disease. Diabetes Obes Metab, 2018. 20 Suppl 1: p. 5-21.

4. Holst, J.J., The incretin system in healthy humans: The role of GIP and GLP-1. Metabolism, 2019. 96: p. 46-55.

5. Gasbjerg, L.S., et al., Separate and Combined Glucometabolic Effects of Endogenous GlucoseDependent Insulinotropic Polypeptide and Glucagon-like Peptide 1 in Healthy Individuals. Diabetes, 2019. 68(5): p. 906-917.

6. Nauck, M.A., et al., Preserved incretin activity of glucagon-like peptide 1 [7-36 amide] but not of synthetic human gastric inhibitory polypeptide in patients with type-2 diabetes mellitus. J Clin Invest, 1993. 91(1): p. 301-7.

7. Calanna, S., et al., Secretion of glucagon-like peptide-1 in patients with type 2 diabetes mellitus: systematic review and meta-analyses of clinical studies. Diabetologia, 2013. 56(5): p. 965-72.

8. Nauck, M.A., et al., Normalization of fasting hyperglycaemia by exogenous glucagon-like peptide 1 (7-36 amide) in type 2 (non-insulin-dependent) diabetic patients. Diabetologia, 1993. 36(8): p. 741-4.

9. Burcelin, R. and P. Gourdy, Harnessing glucagon-like peptide-1 receptor agonists for the pharmacological treatment of overweight and obesity. Obes Rev, 2017. 18(1): p. 86-98.

10. Hare, K.J., et al., The glucagonostatic and insulinotropic effects of glucagon-like peptide 1 contribute equally to its glucose-lowering action. Diabetes, 2010. 59(7): p. 1765-70.

11. Drucker, D.J., J.F. Habener, and J.J. Holst, Discovery, characterization, and clinical development of the glucagon-like peptides. J Clin Invest, 2017. 127(12): p. 4217-4227.

12. Nauck, M.A. and J.J. Meier, MANAGEMENT OF ENDOCRINE DISEASE: Are all GLP-1 agonists equal in the treatment of type 2 diabetes? Eur J Endocrinol, 2019. 181(6): p. R211-R234.

13. Gimeno, R.E., D.A. Briere, and R.J. Seeley, Leveraging the Gut to Treat Metabolic Disease. Cell Metab, 2020.

14. Ferrannini, E. and G. Mingrone, Impact of different bariatric surgical procedures on insulin action and beta-cell function in type 2 diabetes. Diabetes Care, 2009. 32(3): p. 514-20.

15. Jacobsen, S.H., et al., Changes in gastrointestinal hormone responses, insulin sensitivity, and beta-cell function within 2 weeks after gastric bypass in non-diabetic subjects. Obes Surg, 2012. 22(7): p. 1084-96.

16. Mingrone, G., et al., Bariatric surgery versus conventional medical therapy for type 2 diabetes. N Engl J Med, 2012. 366(17): p. 1577-85.

17. Rubino, F., et al., The mechanism of diabetes control after gastrointestinal bypass surgery reveals a role of the proximal small intestine in the pathophysiology of type 2 diabetes. Ann Surg, 2006. 244(5): p. 741-9.

18. Zervos, E.E., et al., Amelioration of insulin requirement in patients undergoing duodenal bypass for reasons other than obesity implicates foregut factors in the pathophysiology of type II diabetes. J Am Coll Surg, 2010. 210(5): p. 564-72, 572-4.

19. Seeley, R.J., A.P. Chambers, and D.A. Sandoval, The role of gut adaptation in the potent effects of multiple bariatric surgeries on obesity and diabetes. Cell Metab, 2015. 21(3): p. 369-78.

20. Salehi, M., R.L. Prigeon, and D.A. D'Alessio, Gastric bypass surgery enhances glucagon-like peptide 1-stimulated postprandial insulin secretion in humans. Diabetes, 2011. 60(9): p. 2308-14. 
21. Larraufie, P., et al., Important Role of the GLP-1 Axis for Glucose Homeostasis after Bariatric Surgery. Cell Rep, 2019. 26(6): p. 1399-1408 e6.

22. Rubino, F., et al., The early effect of the Roux-en-Y gastric bypass on hormones involved in body weight regulation and glucose metabolism. Ann Surg, 2004. 240(2): p. 236-42.

23. Xiong, S.W., et al., Effect of Modified Roux-en-Y Gastric Bypass Surgery on GLP-1, GIP in Patients with Type 2 Diabetes Mellitus. Gastroenterol Res Pract, 2015. 2015: p. 625196.

24. Jorgensen, N.B., et al., Acute and long-term effects of Roux-en-Y gastric bypass on glucose metabolism in subjects with Type 2 diabetes and normal glucose tolerance. Am J Physiol Endocrinol Metab, 2012. 303(1): p. E122-31.

25. Laferrere, B., et al., Effect of weight loss by gastric bypass surgery versus hypocaloric diet on glucose and incretin levels in patients with type 2 diabetes. J Clin Endocrinol Metab, 2008. 93(7): p. 2479-85.

26. Killion, E.A., et al., Glucose-Dependent Insulinotropic Polypeptide Receptor Therapies for the Treatment of Obesity, Do Agonists = Antagonists? Endocr Rev, 2020. 41(1).

27. Yip, R.G., et al., Functional GIP receptors are present on adipocytes. Endocrinology, 1998. 139(9): p. 4004-7.

28. Paratore, S., et al., Gastric inhibitory polypeptide and its receptor are expressed in the central nervous system and support neuronal survival. Cent Nerv Syst Agents Med Chem, 2011. 11(3): p. 210-22.

29. Kim, S.J., C. Nian, and C.H. McIntosh, Activation of lipoprotein lipase by glucose-dependent insulinotropic polypeptide in adipocytes. A role for a protein kinase B, LKB1, and AMPactivated protein kinase cascade. J Biol Chem, 2007. 282(12): p. 8557-67.

30. Asmar, M., et al., Glucose-dependent insulinotropic polypeptide may enhance fatty acid reesterification in subcutaneous abdominal adipose tissue in lean humans. Diabetes, 2010. 59(9): p. 2160-3.

31. Irwin, N., et al., Blockade of gastric inhibitory polypeptide (GIP) action as a novel means of countering insulin resistance in the treatment of obesity-diabetes. Peptides, 2020. 125: $\mathrm{p}$. 170203.

32. Gogebakan, O., et al., Glucose-dependent insulinotropic polypeptide reduces fat-specific expression and activity of 11beta-hydroxysteroid dehydrogenase type 1 and inhibits release of free fatty acids. Diabetes, 2012. 61(2): p. 292-300.

33. Berndt, S.I., et al., Genome-wide meta-analysis identifies 11 new loci for anthropometric traits and provides insights into genetic architecture. Nat Genet, 2013. 45(5): p. 501-12.

34. Turcot, V., et al., Protein-altering variants associated with body mass index implicate pathways that control energy intake and expenditure in obesity. Nat Genet, 2018. 50(1): p. 26-41.

35. Speliotes, E.K., et al., Association analyses of 249,796 individuals reveal 18 new loci associated with body mass index. Nat Genet, 2010. 42(11): p. 937-48.

36. Graff, M., et al., Genome-wide physical activity interactions in adiposity - A meta-analysis of 200,452 adults. PLoS Genet, 2017. 13(4): p. e1006528.

37. Winkler, T.W., et al., The Influence of Age and Sex on Genetic Associations with Adult Body Size and Shape: A Large-Scale Genome-Wide Interaction Study. PLoS Genet, 2015. 11(10): p. e1005378.

38. Bailey, C.J., et al., Immunoreactive gastric inhibitory polypeptide and $K$ cell hyperplasia in obese hyperglycaemic (ob/ob) mice fed high fat and high carbohydrate cafeteria diets. Acta Endocrinol (Copenh), 1986. 112(2): p. 224-9.

39. Adachi, T., et al., Morphological changes and increased sucrase and isomaltase activity in small intestines of insulin-deficient and type 2 diabetic rats. Endocr J, 2003. 50(3): p. 271-9.

40. Miyawaki, K., et al., Inhibition of gastric inhibitory polypeptide signaling prevents obesity. Nat Med, 2002. 8(7): p. 738-42. 
41. Althage, M.C., et al., Targeted ablation of glucose-dependent insulinotropic polypeptideproducing cells in transgenic mice reduces obesity and insulin resistance induced by a high fat diet. J Biol Chem, 2008. 283(26): p. 18365-76.

42. Nasteska, D., et al., Chronic reduction of GIP secretion alleviates obesity and insulin resistance under high-fat diet conditions. Diabetes, 2014. 63(7): p. 2332-43.

43. Gasbjerg, L.S., et al., Glucose-dependent insulinotropic polypeptide (GIP) receptor antagonists as anti-diabetic agents. Peptides, 2018. 100: p. 173-181.

44. Fulurija, A., et al., Vaccination against GIP for the treatment of obesity. PLoS One, 2008. 3(9): p. e3163.

45. Irwin, N., et al., Active immunisation against gastric inhibitory polypeptide (GIP) improves blood glucose control in an animal model of obesity-diabetes. Biol Chem, 2009. 390(1): p. 7580.

46. Boylan, M.O., et al., Gastric inhibitory polypeptide immunoneutralization attenuates development of obesity in mice. Am J Physiol Endocrinol Metab, 2015. 309(12): p. E1008-18.

47. Ravn, P., et al., Structural and pharmacological characterization of novel potent and selective monoclonal antibody antagonists of glucose-dependent insulinotropic polypeptide receptor. J Biol Chem, 2013. 288(27): p. 19760-72.

48. Killion, E.A., et al., Anti-obesity effects of GIPR antagonists alone and in combination with GLP-1R agonists in preclinical models. Sci Transl Med, 2018. 10(472).

49. Pathak, V., et al., Antagonism of gastric inhibitory polypeptide (GIP) by palmitoylation of GIP analogues with $\mathrm{N}$ - and C-terminal modifications improves obesity and metabolic control in high fat fed mice. Mol Cell Endocrinol, 2015. 401: p. 120-9.

50. Hansen, L.S., et al., $N$-terminally and C-terminally truncated forms of glucose-dependent insulinotropic polypeptide are high-affinity competitive antagonists of the human GIP receptor. Br J Pharmacol, 2016. 173(5): p. 826-38.

51. Perry, R.A., et al., Characterisation of Glucose-Dependent Insulinotropic Polypeptide Receptor Antagonists in Rodent Pancreatic Beta Cells and Mice. Clin Med Insights Endocrinol Diabetes, 2019. 12: p. 1179551419875453.

52. Kerr, B.D., et al., Characterization and biological actions of $\mathrm{N}$-terminal truncated forms of glucose-dependent insulinotropic polypeptide. Biochem Biophys Res Commun, 2011. 404(3): p. 870-6.

53. Kaneko, K., et al., Gut-derived GIP activates central Rap1 to impair neural leptin sensitivity during overnutrition. J Clin Invest, 2019. 129(9): p. 3786-3791.

54. Svendsen, B., et al., Pharmacological antagonism of the incretin system protects against dietinduced obesity. Mol Metab, 2020. 32: p. 44-55.

55. Sparre-Ulrich, A.H., et al., GIP(3-30)NH2 is a potent competitive antagonist of the GIP receptor and effectively inhibits GIP-mediated insulin, glucagon, and somatostatin release. Biochem Pharmacol, 2017. 131: p. 78-88.

56. Gasbjerg, L.S., et al., GIP(3-30)NH2 is an efficacious GIP receptor antagonist in humans: a randomised, double-blinded, placebo-controlled, crossover study. Diabetologia, 2018. 61(2): p. 413-423.

57. Baldassano, S., et al., Increased Body Weight and Fat Mass After Subchronic GIP Receptor Antagonist, but Not GLP-2 Receptor Antagonist, Administration in Rats. Front Endocrinol (Lausanne), 2019. 10: p. 492.

58. Mroz, P.A., et al., Optimized GIP analogs promote body weight lowering in mice through GIPR agonism not antagonism. Mol Metab, 2019. 20: p. 51-62.

59. Campbell, J.E., et al., TCF1 links GIPR signaling to the control of beta cell function and survival. Nat Med, 2016. 22(1): p. 84-90.

60. Pamir, N., et al., Glucose-dependent insulinotropic polypeptide receptor null mice exhibit compensatory changes in the enteroinsular axis. Am J Physiol Endocrinol Metab, 2003. 284(5): p. E931-9. 
61. Bailey, C.J., GIP analogues and the treatment of obesity-diabetes. Peptides, 2020. 125: p. 170202.

62. Brandt, S.J., et al., Gut hormone polyagonists for the treatment of type 2 diabetes. Peptides, 2018. 100: p. 190-201.

63. Bastin, M. and F. Andreelli, Dual GIP-GLP1-Receptor Agonists In The Treatment Of Type 2 Diabetes: A Short Review On Emerging Data And Therapeutic Potential. Diabetes Metab Syndr Obes, 2019. 12: p. 1973-1985.

64. Frias, J.P., et al., Efficacy and safety of LY3298176, a novel dual GIP and GLP-1 receptor agonist, in patients with type 2 diabetes: a randomised, placebo-controlled and active comparator-controlled phase 2 trial. Lancet, 2018. 392(10160): p. 2180-2193.

65. Coskun, T., et al., LY3298176, a novel dual GIP and GLP-1 receptor agonist for the treatment of type 2 diabetes mellitus: From discovery to clinical proof of concept. Mol Metab, 2018. 18: p. 3-14.

66. Lu, H., et al., Subcutaneous Angiotensin II Infusion using Osmotic Pumps Induces Aortic Aneurysms in Mice. J Vis Exp, 2015(103).

67. Matthews, D.R., et al., Homeostasis model assessment: insulin resistance and beta-cell function from fasting plasma glucose and insulin concentrations in man. Diabetologia, 1985. 28(7): p. 412-9.

68. Nauck, M.A., et al., Cardiovascular Actions and Clinical Outcomes With Glucagon-Like Peptide-1 Receptor Agonists and Dipeptidyl Peptidase-4 Inhibitors. Circulation, 2017. 136(9): p. 849-870.

69. Chukir, T., et al., Pharmacotherapy for obesity in individuals with type 2 diabetes. Expert Opin Pharmacother, 2018. 19(3): p. 223-231.

70. Filippatos, T.D., T.V. Panagiotopoulou, and M.S. Elisaf, Adverse Effects of GLP-1 Receptor Agonists. Rev Diabet Stud, 2014. 11(3-4): p. 202-30.

71. Holst, J.J. and M.M. Rosenkilde, GIP as a therapeutic target in diabetes and obesity: insight from incretin co-agonists. J Clin Endocrinol Metab, 2020.

72. Sparre-Ulrich, A.H., et al., Species-specific action of (Pro3) GIP - a full agonist at human GIP receptors, but a partial agonist and competitive antagonist at rat and mouse GIP receptors. Br J Pharmacol, 2016. 173(1): p. 27-38.

73. Gabe, M.B.N., et al., Human GIP(3-30)NH2 inhibits $G$ protein-dependent as well as $G$ proteinindependent signaling and is selective for the GIP receptor with high-affinity binding to primate but not rodent GIP receptors. Biochem Pharmacol, 2018. 150: p. 97-107. 
not certified by peer review) is the author/funder, who has granted bioRxiv a license to display the preprint in perpetuity. It is made availa under aCC-BY-ND 4.0 International license.

A

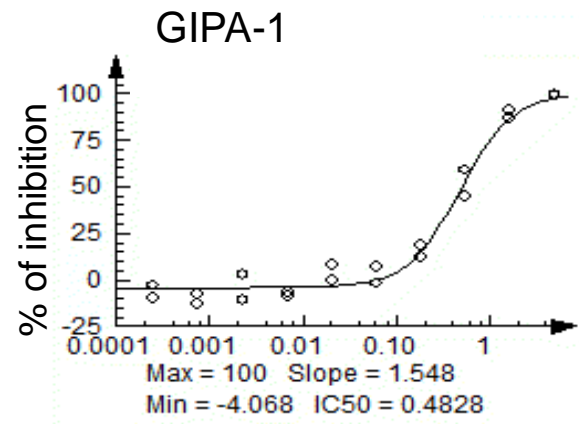

C

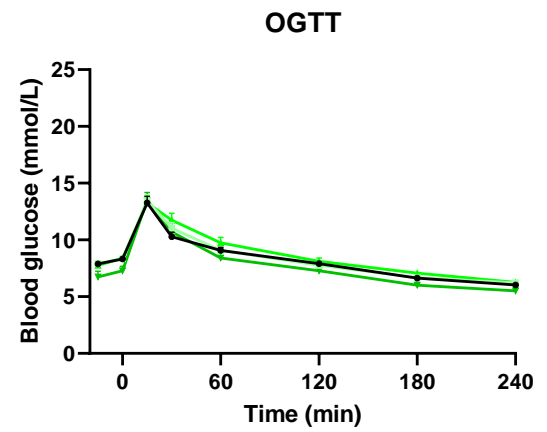

E

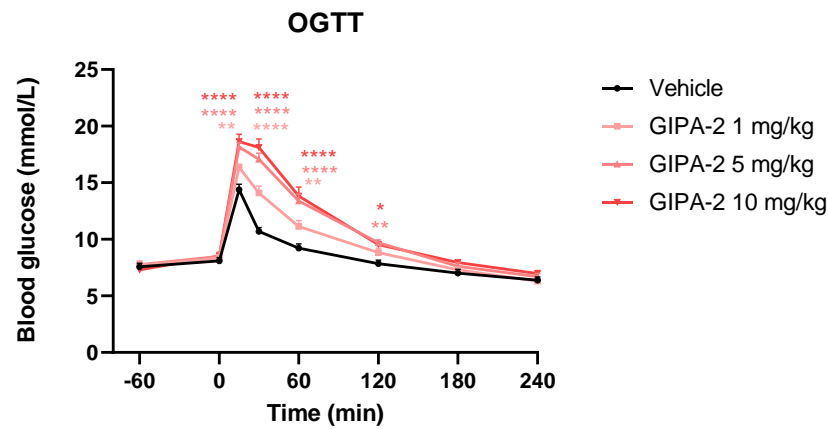

G

Insulin-15 min

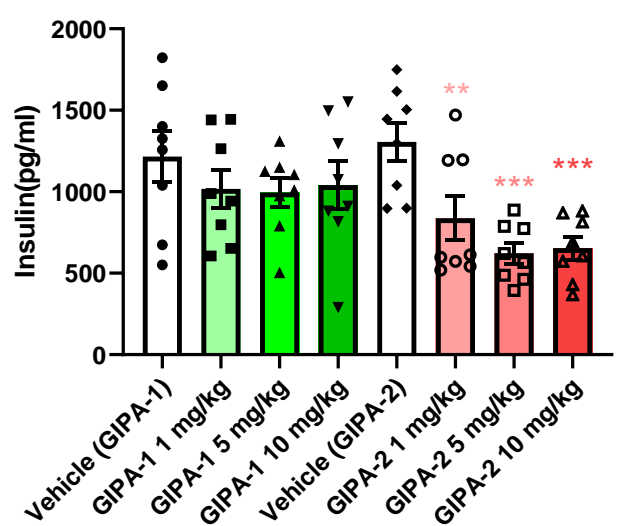

AUC for IPGTT

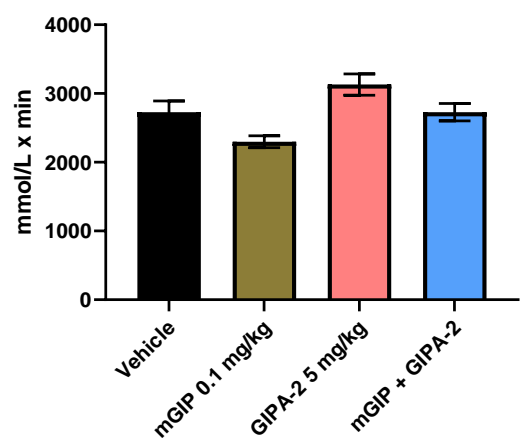

口 GIPA-2 $5 \mathrm{mg} / \mathrm{kg}$

DGIP + GIPA-2
$\mathrm{B}$

GIPA-2

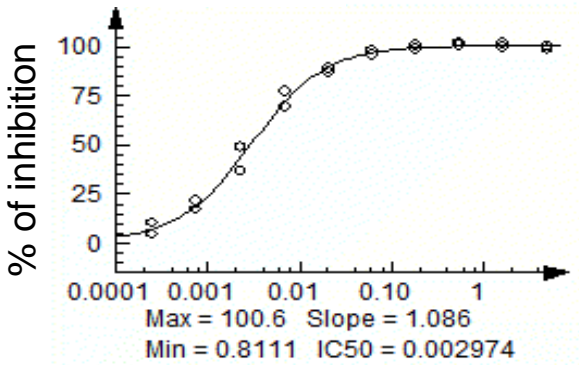

D

$\rightarrow$ Vehicle

- GIPA-1 $1 \mathrm{mg} / \mathrm{kg}$

- GIPA-1 $5 \mathrm{mg} / \mathrm{kg}$

- GIPA-1 $10 \mathrm{mg} / \mathrm{kg}$

F

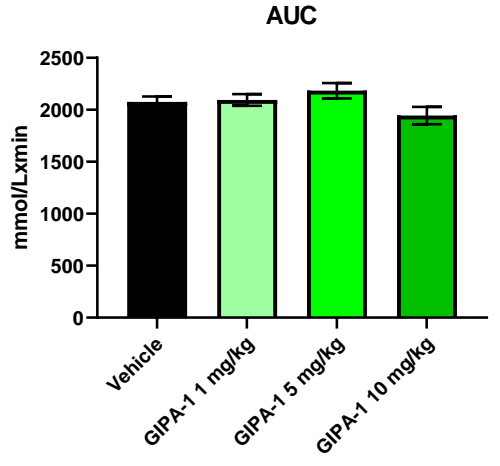

- Vehicle

ㅁ GIPA-1 $1 \mathrm{mg} / \mathrm{kg}$

ㅁ GIPA-1 $5 \mathrm{mg} / \mathrm{kg}$

ㅁ GIPA-1 $10 \mathrm{mg} / \mathrm{kg}$
$\mathrm{H}$

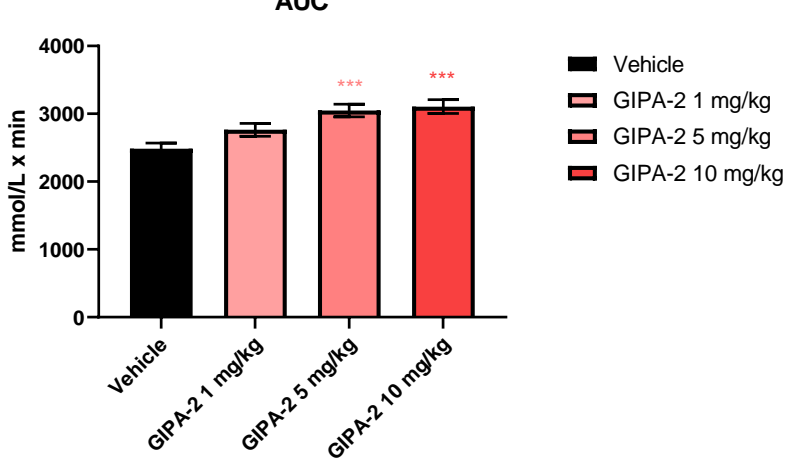

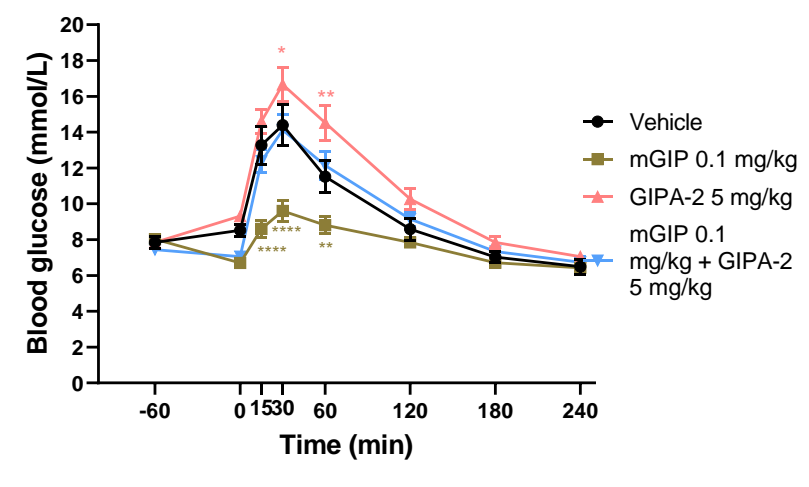

Insulin

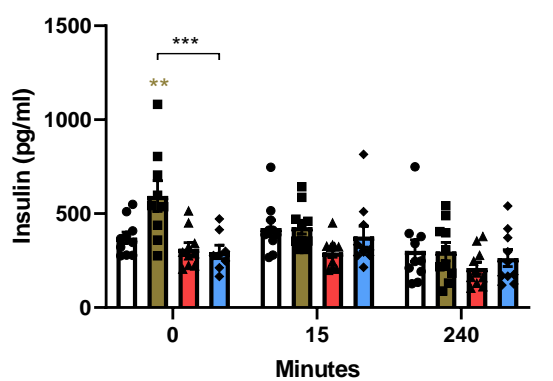

- Vehicle

- $\mathrm{mGIP} 0.1 \mathrm{mg} / \mathrm{kg}$

- GIPA-2 $5 \mathrm{mg} / \mathrm{kg}$

- $m$ GIP $0.1 \mathrm{mg} / \mathrm{kg}$ +

GIPA-2 5 mg/kg 

not certified by peer review) is the author/funder, who has granted bioRxiv a license to display the preprint in perpetuity. It is made availa

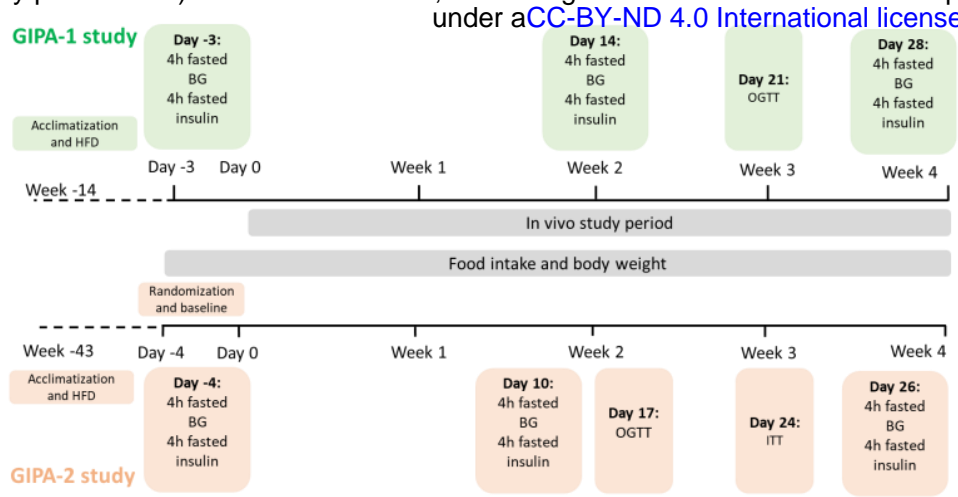

B

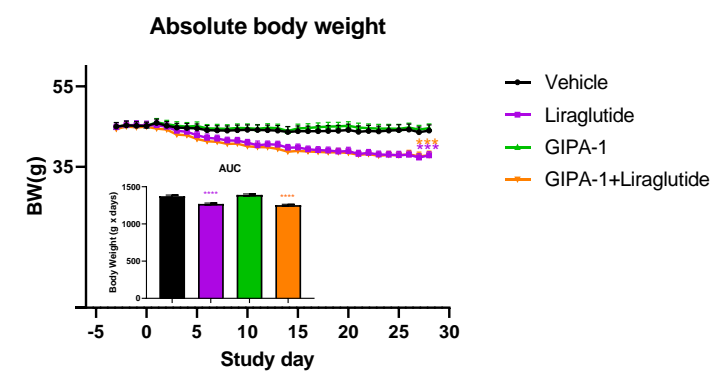

D

F

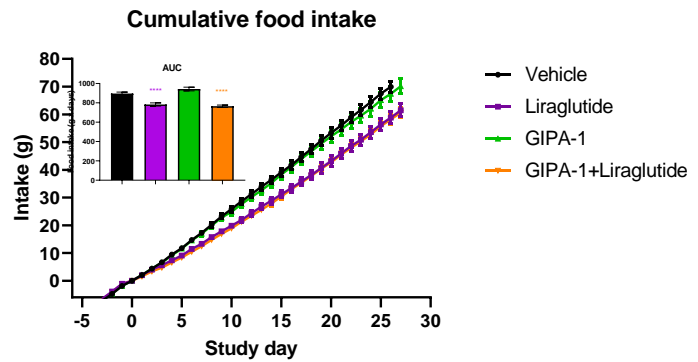

Fasting blood glucose

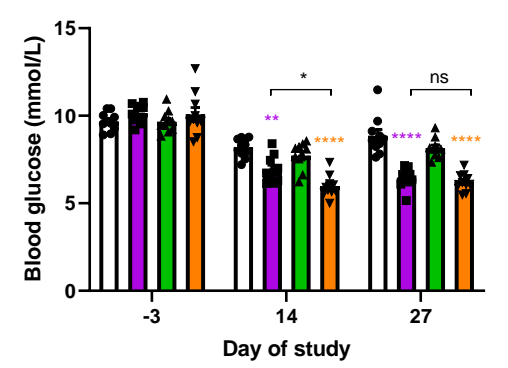

$\mathrm{H}$

Fasting insulin

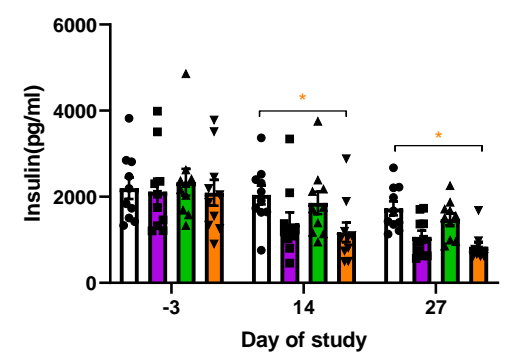

J

HOMA-IR- GIPA-1

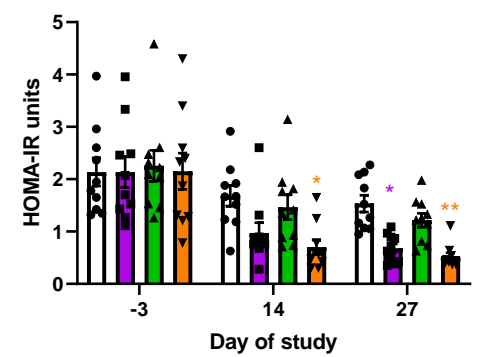

- Vehicle

- Liraglutide

- GIPA-1

- Vehicle

- Liraglutide

- GIPA-1

- Vehicle

- Liraglutide

- GIPA-1
C

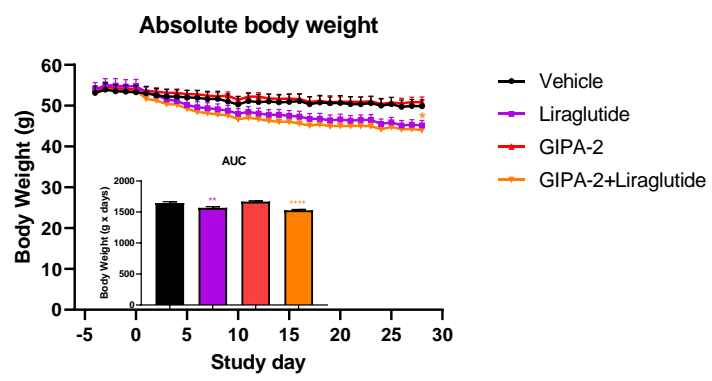

E

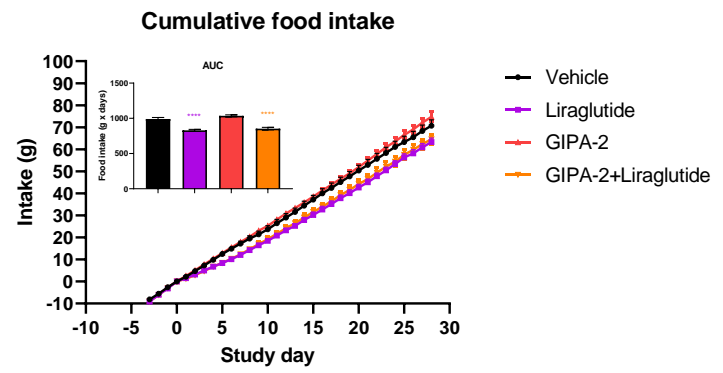

G

- GIPA-1+Liraglutide

- GIPA-1+Liraglutide

- GIPA-1+Liraglutide

K

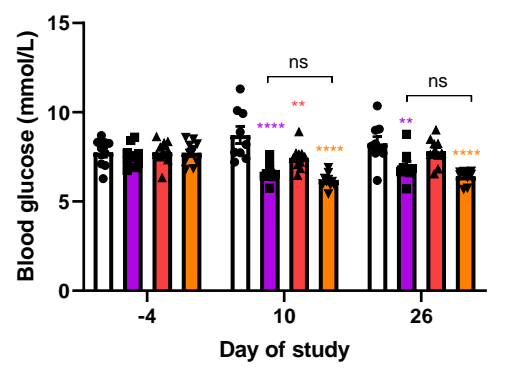

- Vehicle

- Liraglutide

- GIPA-2

- GIPA-2+Liraglutide

Fasting insulin

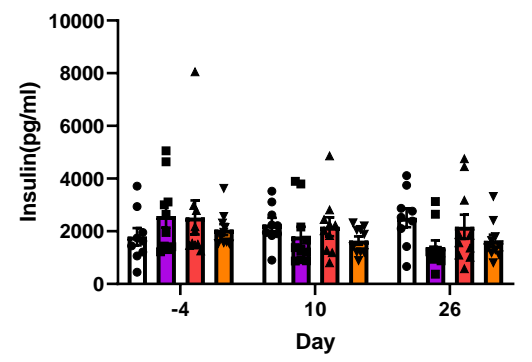

- Vehicle

- Liraglutide

- GIPA-2

- GIPA-2+Liraglutide
HOMA-IR- GIPA-2

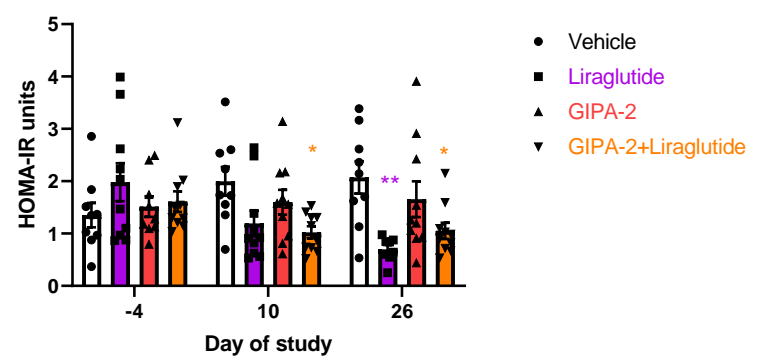


not certified by peer review) is the author/funder, who has granted bioRxiv a license to display the preprint in perpetuity. It is made availa under aCC-BY-ND 4.0 International license.

A

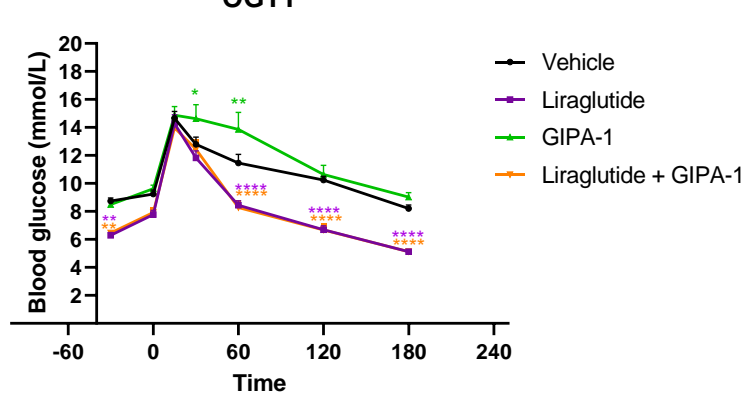

C

E

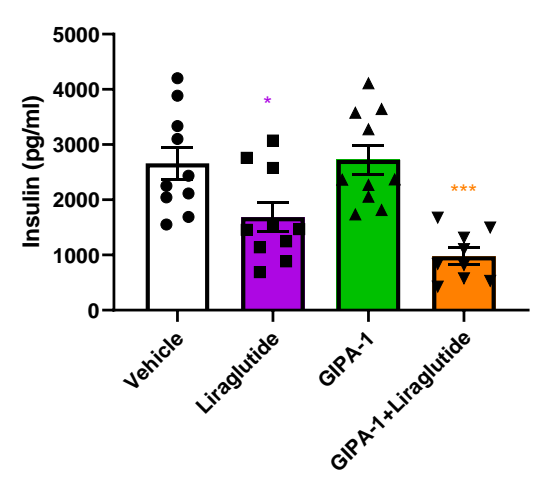

G

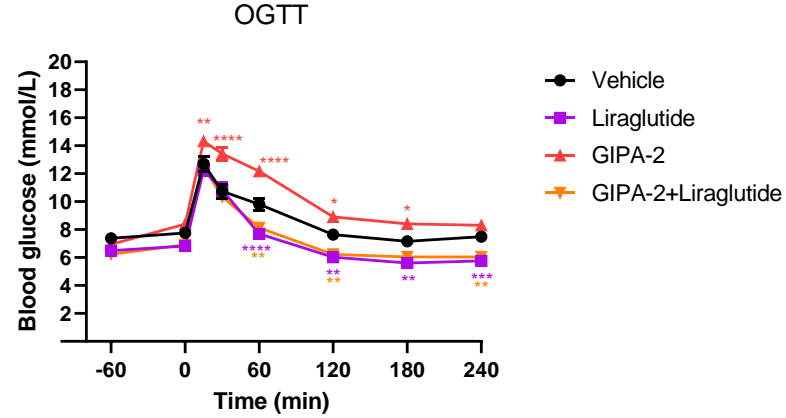

ITT

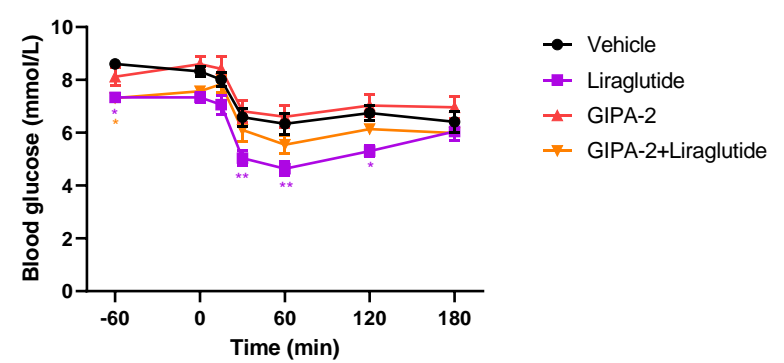

B

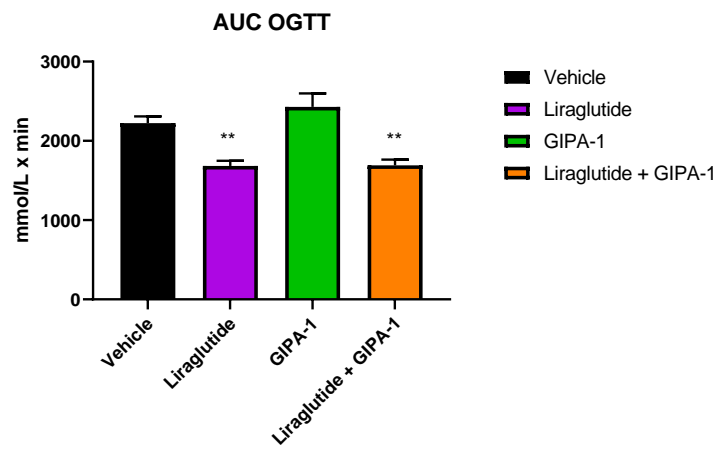

Insulin- Time $15 \mathrm{~min}$

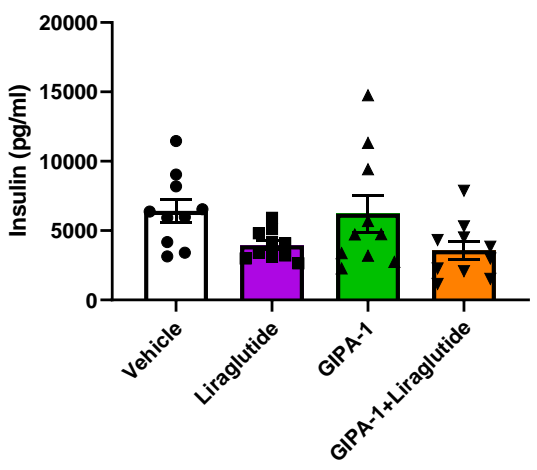

F

$\mathrm{H}$

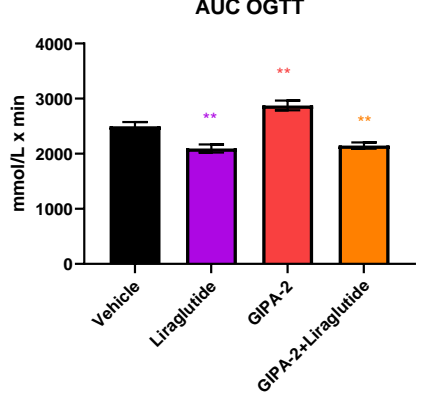

- Vehicle

口 Liraglutide

口 GIPA-2

口 GIPA-2+Liraglutide

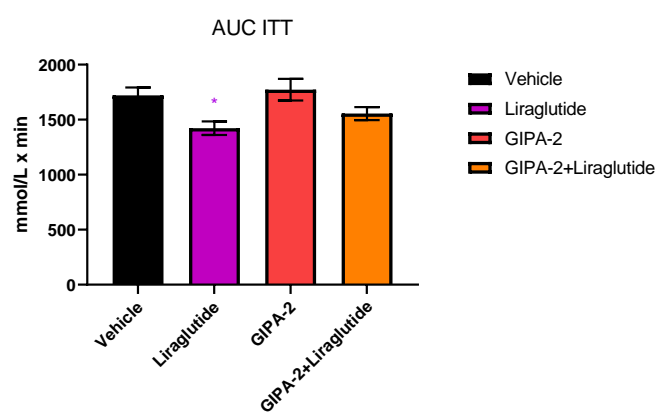


not certified by peer review) is the author/funder, who has granted bioRxiv a license to display the preprint in perpetuity. It is made availa

A

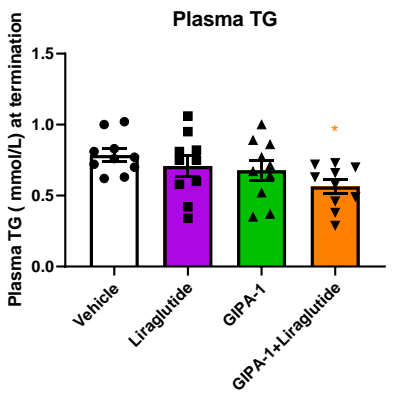

C

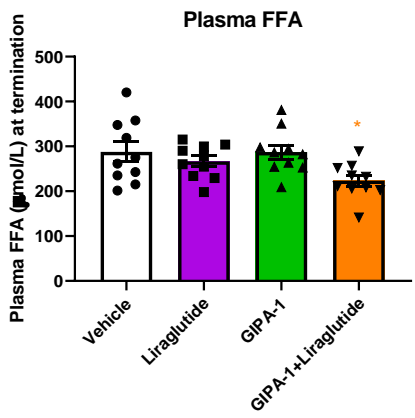

E

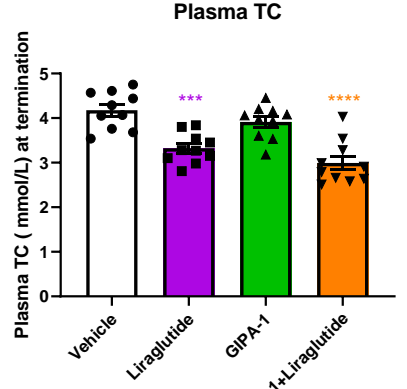

G
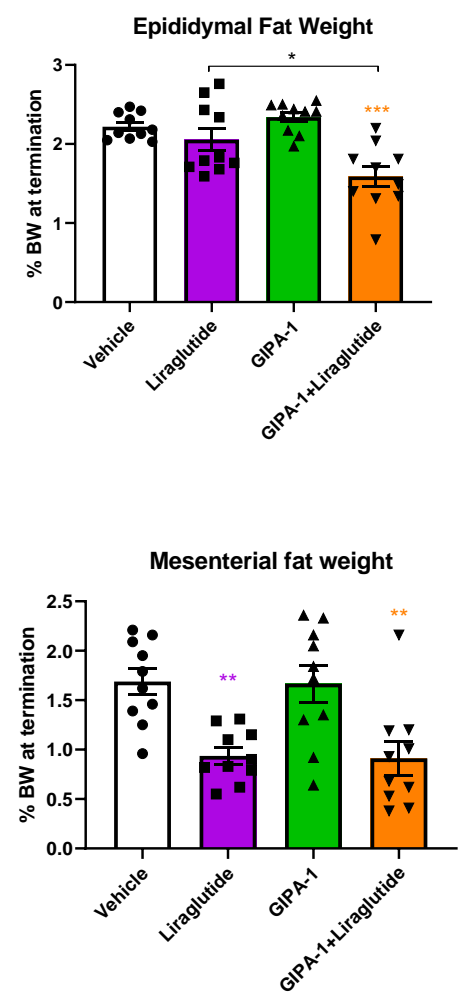

B

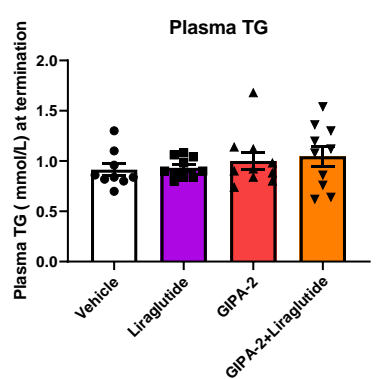

D

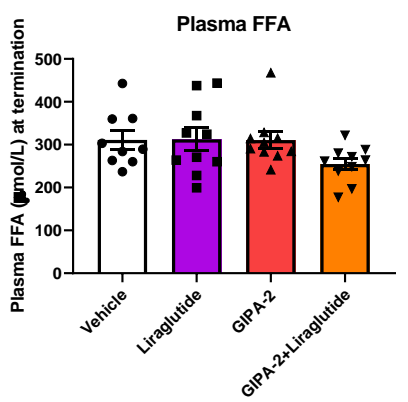

F

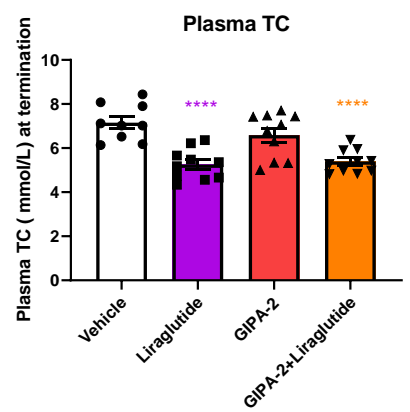

$\mathrm{H}$
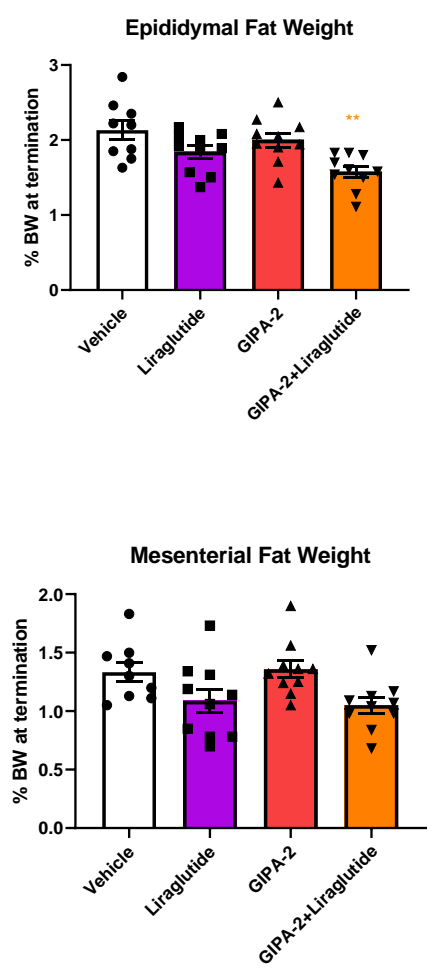